\title{
SPECTROELECTROCHEMISTRY: A SURVEY OF IN SITU SPECTROSCOPIC TECHNIQUES
}

\author{
(Technical Report) \\ Prepared for publication by \\ W. PLIETH ${ }^{1}$, G. S. WILSON ${ }^{2}$ AND C. GUTIÉRREZ DE LA FE ${ }^{3}$ \\ ${ }^{1}$ Institut für Physikalische Chemie und Elektrochemie, Technische Universität Dresden, Bergstr. 66b,
D-01062 Dresden, Germany
${ }^{2}$ Department of Chemistry, University of Kansas, Lawrence, KS 66045, USA
${ }^{3}$ Instituto de Quimica Fisica "Rocasolano", Calle Serrano 119, Madrid 28006, Spain
}

The coordinators acknowledge the contributions of H.-H. Strehblow (Germany) and L. Dunsch (Germany).

*Membership of the Commission during the period the report was prepared:

1991-1993: Titular Members: G. S. Wilson (USA, Chairman); W. Plieth (Germany, Secretary); P. Andrieux (France); D. M. Drazic (Yugoslavia); V. M. M. Lobo (Portugal); M. Sluyters-Rehbach (Netherlands); K. Tokuda (Japan); Associate Members: B. E. Conway (Canada); G. Kreysa (Germany); C. F. Martin(USA); A. A. Milchev (Bulgaria); T. Watanabe (Japan); National Representatives; G. Gritzner (Austria); T. Rabockai (Brazil); G. Inzelt (Hungary); S. K. Rangarajan (India); S. Trasatti (Italy); W.-K. Paik (Republic of Korea); C. Gutiérez de la Fe (Spain); D. Simonsson (Sweden); M. L. Berkem (Turkey); 1994-1995: Titular Members: G. S. Wilson (USA, Chairman); W. Plieth (Germany, Secretary); C. P. Andrieux (France); W. J. Lorenz (Germany); M. Sluyters-Rehbach (Netherlands); K. Tokuda (Japan); T. Watanabe (Japan); Associate Members: C. M. A. Brett (Portugal); F. M. Hawkridge (USA); V. E. Kazarinov (Russia); C. R. Martin (USA); National Representatives: V. A. Macagno (Argentina); G. Gritzner (Austria); T. Rabockai (Brazil); G. Inzelt (Hungary); S. K. Rangarajan (India); I. Rubinstein (Israel); S. Trasatti (Italy); W.-K. Paik (Republic of Korea); C. Gutiérrez de la Fe (Spain); D. Simonsson (Sweden); C. A. Vincent (United Kingdom); 1996-1997: Titular Members: W. Plieth (Germany, Chairman); M. Sluyters Rehbach (Netherlands, Secretary); C. P. Andrieux (France); C. M. A. Brett (Potugal); D. J. Schiffrin (United Kingdom); T. Watanabe (Japan); Associate Members: M. de Vogelaere (Germany); F. M. Hawkridge (USA); Z. Samec (Tschechien); H. Siegenthaler (Switzerland); K. Uosaki (Japan); National Representatives: V. A. Macagno (Argentina); G. Gritzner (Austria); Ch. Bai (People Republic of China); G. Inzelt (Hungary); I. Rubinstein (Israel); W.-K. Paik (Republic of Korea); C. Gutiérrez de la Fe (Spain);. K. E Ahlberg (Sweden).

Republication or reproduction of this report or its storage and/or dissemination by electronic means is permitted without the need for formal IUPAC permission on condition that an acknowledgement, with full reference to the source along with use of the copyright symbol $\odot$, the name IUPAC and the year of publication are prominently visible. Publication of a translation into another language is subject to the additional condition of prior approval from the relevant IUPAC National Adhering Organization. 


\section{Spectroelectrochemistry: A survey of in situ spectroscopic techniques (Technical Report)}

Abstract: In this technical paper a summary of the available in situ spectroelectrochemical methods, their basic principles, their typical applications, and their limitations is given. With respect to the names of the methods and usual abbreviations, the paper follows the literature as far as possible, but tries to point out inconsistencies. An introductory section gives a summary of the basic equations and introduces the IUPAC recommendations for quantities and symbols.

\section{CHAPTER}

PAGE

1. INTRODUCTION 1

2. BASIC ASPECTS 2

2.1. The electrode/electrolyte interphase 2

2.2. The optical properties of a homogeneous phase 2

2.3. Transmission and reflection of light 3

2.3.1. Reflection of light 3

2.3.2. Reflection in the Presence of a Surface Film 4

2.3.3. Transmission of light 6

2.3.4. Transmission in the Presence of a Surface Film 6

2.3.5. Consideration of Uniaxial Anisotropy and Nonlinear Optics 7

3. TRANSMISSION EXPERIMENTS

3.1. Optically Transparent Electrodes $\quad 7$

3.2. Long Optical Path Thin Layer Cells 10

3.3. Diffusion Layer Imaging (or Profiling) 11

4. REFLECTANCE EXPERIMENTS 11

4.1. UV/VIS Reflectance Spectroscopy 11

4.1.1. UV/VIS Differential Reflectance Spectroscopy 11

4.1.2. UV/VIS Electrochemically Modulated Reflectance Spectroscopy (or Electrolyte

$\begin{array}{ll}\text { Electroreflectance Spectroscopy) } & 12\end{array}$

4.1.3. UV/VIS Wavelength Modulated Reflectance Spectroscopy 14

4.1.4. UV/VIS Laser Modulated Reflectance Spectroscopy 14

4.1.5. UV/VIS Attenuated Total Reflectance Spectroscopy (or Internal Reflectance Spectroscopy)

4.1.6 UV/VIS Surface Plasmon Spectroscopy $\quad 17$

4.2. Infrared Reflectance Spectroscopy $\quad 16$

4.2.1. Electrochemically Modulated Infrared Reflectance Spectroscopy 16

4.2.2. Differential Fourier Transform Infrared Reflectance Spectroscopy 17

4.2.3. Polarization Modulated Fourier Transform Infrared Reflectance Spectroscopy. 18

4.2.4. Multiple Internal Reflection Fourier Transform Infrared Reflectance Spectroscopy 19

5. METHODS BASED ON THE POLARIZATION OF LIGHT 19

5.1. Ellipsometry 19

5.2. Circular and Linear Dichroism 22

6. METHODS BASED ON SCATTERED LIGHT 22

6.1. Light Scattering from Non-flat Surfaces 22

6.2. Surface Raman Spectroscopy 22 
6.2.1. Surface Enhanced Raman Spectroscopy 23

6.3. Nonlinear Optical Techniques 24

7. X-RAY AND $\gamma$-RAY TECHNIQUES

$7.1 \quad X$-ray Diffraction $\quad 25$

7.2 X-ray Reflection and Diffraction at Grazing Incidence $\quad 26$

7.3 X-ray Standing Wave Fluorescence 26

7.1. EXAFS and Related Techniques $\quad 27$

7.2. Mößbauer Spectroscopy 27

8. MAGNETIC RESONANCE METHODS, MICROWAVE SPECTROSCOPY 28

8.1. Electron Paramagnetic Resonance (EPR, ESR) 28

8.2. Microwave Absorption 28

8.3. Nuclear Magnetic Resonance (NMR) Spectroscopy 29

9. PHOTOCURRENT AND PHOTOPOTENTIAL SPECTROSCOPIES 30

10 PHOTOTHERMAL METHODS 31

10.1. Photoacoustic Spectroscopy (PAS) 31

10.2. Photothermal Probe Beam Deflection Spectroscopy 31

10.3 Probe Beam Deflectometry 31

11. LIGHT EMISSION METHODS 32

11.1. Electrochemical Luminescence $\quad 32$

11.2. Electroluminescence of Semiconductors 32

\section{INTRODUCTION}

This paper gives descriptions and recommendations of spectroelectrochemical in situ methods based primarily on transmission, reflection, and scattering of electromagnetic radiation. The region of electromagnetic radiation covers the wavelength range from radio frequencies to $\gamma$-rays and is defined in an IUPAC document (ref. 1). Additional definitions of optical spectroscopy are found in the same document. Excluded are ex situ methods, which are the subject of a separate IUPAC project (ref. 2). As far as possible the symbols used follow the recommendations of IUPAC's "Green Book" (ref. 3).

\section{BASIC ASPECTS}

\subsection{The electrode/electrolyte interphase}

The electrode/electrolyte interphase consists of discrete or overlapping domains which may contribute to the spectroelectrochemical response. These domains would include

- the bulk of the electrode;

- the surface layer, influenced by the electric field: i) of the free electron gas (Thomas-Fermi layer); ii) of surface states; iii) of the bound electrons, if the electrode is a metal;

- the space charge layer and surface states, if the electrode is a semiconductor;

- the electrochemical double layer, consisting of an inner (Helmholtz) and a diffuse (Gouy-Chapman) layer;

- organic or ionic adsorbed species within the electrochemical double layer;

- multimolecular, inorganic and organic films;

- $\quad$ any films formed by reaction of the electrode with the environment (for example, oxide films);

- transport layers under hydrodynamic flow conditions, such as diffusion layers etc.;

- homogeneous reaction layers;

- migration or convection layers (if driving forces in addition to chemical potential gradients (diffusion) act on the transport processes); 
- $\quad$ the bulk electrolyte.

A frequently encountered simplification is the three-layer model, consisting of the bulk electrode, one intermediate layer, and the bulk electrolyte. More complex models take into account optical anisotropy or, in the case of the double layer, the smooth transition between the two media (transition layer model, Section 2.3.5).

\subsection{The optical properties of a homogeneous phase}

The optical properties of a homogeneous phase are described by a complex refractive index, $\hat{n}$ consisting of a refractive index, $n$, and an absorption index, $k$, and defined as

$\hat{n}=n-\mathrm{i} k$

according to the Nebraska convention (ref. 4), where $i$ is defined as $\sqrt{-1}$. Instead of the complex refractive index, $\hat{n}$, the complex relative dielectric permittivity, $\hat{\varepsilon}_{r}$ can be used

$\hat{\varepsilon}_{r}=\hat{n}^{2}=\varepsilon_{r}^{\prime}-\mathrm{i} \varepsilon_{r}^{\prime \prime}$

$\varepsilon_{r}^{\prime}$ and $\varepsilon_{r}^{\prime \prime}$ are related to the real and imaginary parts of $\hat{n}$ by the equations

$\varepsilon_{r}^{\prime}=n^{2}-k^{2}$

$\varepsilon_{r}^{\prime \prime}=2 n k$

provided that the relative magnetic permeability, $\mu_{r}$, is unity.

\subsection{Transmission and reflection of light}

If an electromagnetic wave encounters a phase boundary (between phases $j$ and $k$, Fig. 1), it is partially transmitted and partially reflected. The classical description of the transmitted and the reflected electromagnetic wave is given by the Fresnel theory (ref. 5). It is assumed that the two semi-infinite media are homogeneous and optically isotropic, and that reflection takes place at the planar interface. The medium $j$ (e.g., transparent bulk electrolyte) is characterized by the refractive index, $n_{j}$ and the absorbing substrate $k$ (e.g., metal) by the complex refractive index, $\hat{n}_{k}$. The angle of incidence, $\alpha_{j}$, is related to the complex angle of refraction, $\hat{\alpha}_{k}$, by Snell's (Descarte's) law: $n_{j} \sin \alpha_{j}=\hat{n}_{k} \sin \hat{\alpha}_{k}$

\subsubsection{Reflection of light}

The electromagnetic wave reflected from the interface between phase $j$ and phase $k$ is usually described by the Fresnel reflection coefficient, which is the ratio of the complex amplitudes of the electrical components of the reflected (refl) and incident (o) electromagnetic waves,

$\hat{r}_{j k}=\frac{E_{\text {refl }}}{E_{0}} \exp \left[\mathrm{i}\left(\phi_{\text {refl }}-\phi_{0}\right)\right]$

where $E_{\text {refl and }} E_{0}$ are the amplitudes, and $\phi_{\text {refl and }} \phi_{0}$ the phases, of the reflected and incident electromagnetic waves, respectively. For light polarized perpendicular ( $s$ ) or parallel $(\mathrm{p})$ to the plane of incidence the Fresnel reflection coefficient is :

$\hat{r}_{j k, \mathrm{~s}}=\frac{n_{j} \cos \alpha_{j}-\hat{n}_{k} \cos \hat{\alpha}_{k}}{n_{j} \cos \alpha_{j}+\hat{n}_{k} \cos \hat{\alpha}_{k}}$ 


$$
\hat{r}_{j k, \mathrm{p}}=\frac{\hat{n}_{k} \cos \alpha_{j}-n_{j} \cos \hat{\alpha}_{k}}{\hat{n}_{k} \cos \alpha_{j}+n_{j} \cos \hat{\alpha}_{k}}
$$

respectively.

The reflectance, $\rho$, is the ratio of the reflected, $I_{\text {refl }}$, to the incident, $I_{0}$, intensity of the light, and is equal to the product of $\hat{r}_{j k}$ and its complex conjugate, $\hat{r}_{j k}^{*}$.

$$
\rho_{j k}=\frac{I_{\text {refl }}}{I_{0}}=\hat{r}_{j k} \hat{r}_{j k}^{*}
$$

\subsubsection{Reflection in the Presence of a Surface Film}

In the three-layer model a parallel-sided absorbing film (phase 2) between phase 1 (e. g. water) and phase 3 (e. g. metal electrode) is assumed to be homogeneous and optically isotropic with the refractive index, $\hat{n}_{2}$, and a uniform thickness, $d$. For this system Drude's equations for the total reflection coefficient $\hat{r}_{123}$ are (ref. 6):

$$
\hat{r}_{123, i}=\frac{\hat{r}_{12, i}+\hat{r}_{23, i} \exp (-2 \mathrm{i} \beta)}{1+\hat{r}_{12, i} r_{23, i} \exp (-2 \mathrm{i} \beta)}
$$

with $i$ equal to $\mathrm{s}$ or $\mathrm{p}$, respectively, for light polarized perpendicular or parallel to the plane of incidence, respectively, and

$$
\beta=2 \pi \frac{d}{\lambda} n_{2} \cos \alpha
$$

where $\lambda$ is the wavelength of the incident light. The interfacial reflection coefficients $\hat{r}_{j k}$ are given by eqs. 6 and 7, where $j, k=1,2$ or 2,3, and the angles $a_{j}$ and $\hat{a}_{k}$ are related by Snell's law.

The three-layer model has been applied to the electrochemical double layer (ref. 7). When the film thickness is much smaller than the wavelength $(d / \lambda<<1)$, it is possible to linearize eq. (9).

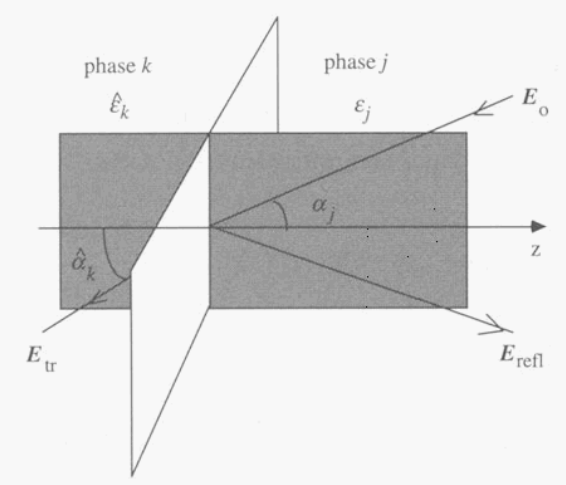

Fig. 1. Reflection and transmission of light at a smooth interface between two homogeneous phases $j$ (transparent) and $k$ (absorbing). $E_{0}, E_{\text {refl }}, E_{\mathrm{tr}}$ : incident, reflected, transmitted electromagnetic waves, respectively; $\varepsilon_{j}, \alpha_{j}, \hat{\varepsilon}_{k}, \hat{\alpha}_{k}$ : relative dielectric permittivity and the angle of the light beam with the z-axis nomnal to the surface in phases $j$ and $k$, respectively (in the absorbing phase $k$ both $\varepsilon$ and $\alpha$ are complex); the grey area is the plane of incidence, defined by $E_{0}, E_{\text {refl }}$ and $E_{\mathrm{tr}}$. 


\subsubsection{Transmission of light}

The transmitted electromagnetic wave is described by the Fresnel transmission coefficient, which is the ratio of the complex amplitudes of the electrical component of the transmitted (tr) to that of the incident (o) electromagnetic wave,

$\hat{t}_{j k}=\frac{\boldsymbol{E}_{\mathrm{tr}}}{\boldsymbol{E}_{0}} \exp \left[\mathrm{i}\left(\phi_{\mathrm{tr}}-\phi_{0}\right)\right]$

where $E_{\mathrm{tr}}$ and $E_{0}$ are the amplitudes, and $\phi_{\mathrm{tr}}$ and $\phi_{0}$ the phases, of the transmitted and incident electromagnetic waves, respectively. For light polarized perpendicular (s) or parallel (p) to the plane of incidence the Fresnel transmission coefficient, $\hat{t}_{j k}$, is :

$\hat{t}_{j k, \mathrm{~s}}=\frac{2 n_{j} \cos \alpha_{j}}{n_{j} \cos \alpha_{j}+\hat{n}_{k} \cos \hat{\alpha}_{k}}$

$\hat{t}_{j k, \mathrm{p}}=\frac{2 \hat{n}_{k} \cos \alpha_{j}}{\hat{n}_{k} \cos \alpha_{j}+n_{j} \cos \hat{\alpha}_{k}}$

respectively.

\subsubsection{Transmission in the Presence of a Surface Film}

Again, a parallel-sided absorbing film (phase 2) between phase 1 and phase 3 is assumed to be homogeneous and optically isotropic with a refractive index, $\hat{n}_{2}$, and a uniform thickness, $d$ For the three-layer system the transmission coefficient, $\hat{t}_{123, i}$, is:

$\hat{t}_{123, i}=\frac{\hat{t}_{12, i} \cdot \hat{t}_{23, i} \exp (-\mathrm{i} \beta)}{1+\hat{r}_{12, i} \cdot \hat{r}_{23, i} \exp (-2 \mathrm{i} \beta)}$

with $\beta$ given again by eq. 10. The subindex $i$ is equal to $\mathrm{s}$ and $\mathrm{p}$ for polarization perpendicular and parallel, respectively, to the plane of incidence.

\subsubsection{Consideration of Uniaxial Anisotropy and Nonlinear Optics}

The above equations can be extended to the case of a film with uniaxial anisotropy whose dielectric permittivity is represented by a tensor with two components, $\varepsilon_{\mathrm{t}}$ and $\varepsilon_{\mathrm{n}}$, tangential and normal to the surface, respectively (ref. 8-10).

For some metals like silver and gold, nonlinear optical effects must be considered (ref. 11).

\section{TRANSMISSION EXPERIMENTS}

Transmission methods are based on the measurement of the decrease of intensity, $I$, of a light beam after interaction with the electrochemical cell. The method is restricted to the wavelength region in which the electrolyte is transparent (in the case of water UV radiation above $200 \mathrm{~nm}$ and visible light). Conservation of energy demands that the absorptance, $\alpha=I_{\mathrm{abs}} / I_{0}$, transmittance, $\tau=I_{\mathrm{tr}} / I_{0}$, reflectance, $\rho=I_{\text {refl }} / I_{0}$ and scattering, $\sigma=I_{\text {scat }} / I_{0}$ add up to unity: $\alpha+\tau+\rho+\sigma=1$. If the reflected and scattered intensities are small compared to the absorbed intensities, the absorptance is $\alpha=1-\tau$. The quantity usually measured is the absorbance, $A=-\log (1-\alpha)$ ). Usually the light beam is perpendicular to the electrode surface, although in some techniques it is parallel. 


\subsection{Optically Transparent Electrodes}

Optically transparent electrodes (OTE) can be used to construct optically transparent cells which can be used in a conventional UV/VIS spectrometer (ref. 12-14). Optically transparent thin layer electrochemical cells (OTTLE) are a useful variant. The optically transparent electrode can be:

- a thin metal film on a transparent substrate. The thickness must not exceed $100 \mathrm{~nm}$ for the film to remain transparent, and this can result in high electrical resistance.

- a glass plate with a thin film of an optically transparent, conducting material, for example, indiumdoped tin oxide (ITO);

- a gold minigrid between transparent substrates;

- a thicker, free-standing metal mesh.

The optically transparent electrode is used as a single working electrode or as a stack of working electrodes and is combined with an auxiliary electrode and a reference electrode in a spectroelectrochemical cell (Fig. 2). One measures the transmitted intensity as a function of the potential $U$. In OTTLEs large potential drops can occur in the thin layer cell due to its high solution resistance, resulting in non-uniform potential and product distributions across the electrode surface. Typical plots show

a) absorptance, $\alpha$ as a function of potential at a constant wavelength, $\alpha=f(U)_{\lambda}$ (absorptogram).

b) absorptance, $\alpha$, as a function of the wavelength, $\alpha=f(\lambda)_{U}$ at a constant potential. This is the absorption spectrum of the solution or surface film,

c) absorptance, $\alpha$, as a function of time after a potential perturbation, usually a potential step, $\alpha=f(t)_{\lambda}$ (chronoabsorptometry) Alternatively, a potential scan is used (voltabsorptometry).

The derivative of the signal with respect to time has the shape of a cyclic voltammogram* (ref. 15).

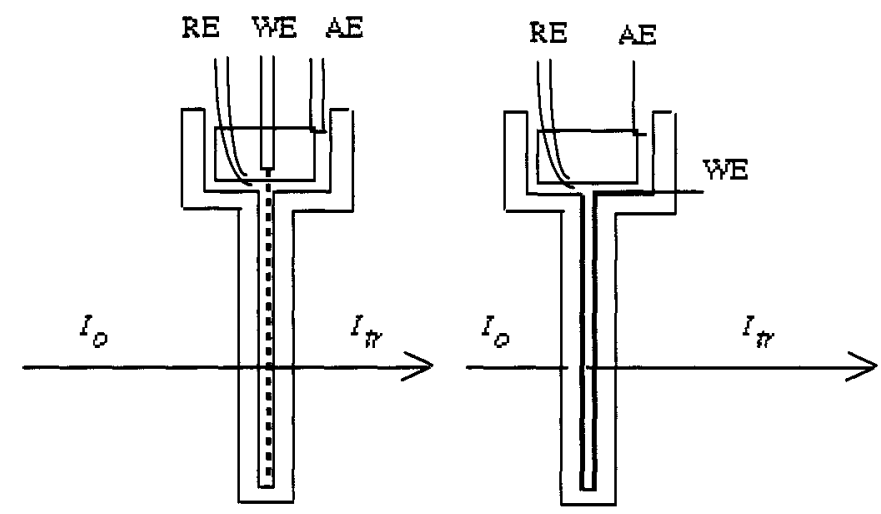

Fig. 2. Optically transparent electrochemical cells: a) minigrid as WE, b) evaporated cell wall as WE. $I_{0}, I_{\mathrm{tr}}$, incident and transmitted intensity; WE, RE, and AE: working, reference and auxiliary electrode, respectively.

The background absorption is mostly originated by the metal film or grid. Division of the transmitted intensity $I_{\mathrm{tr}}(U)$ by the background value $I_{0}$ (preferentially at a suitable reference potential), yields the transmittance, $\tau(U)=I_{\mathrm{tr}}(U) / I_{0}$ from which one calculates the absorptance, $\alpha(U)=1-\tau(U)$.

Although in principle the true absorption index, $\mathrm{k}$, of the interface layer should be calculated because potential-dependent interference effects may influence the results, this complication is generally neglected.

Ideally, when using OTEs the reactant (dissolved in bulk solution) should not absorb radiation in the wavelength window of the product. If it does, detecting the product may be difficult if the beam traverses a significant thickness of reactant solution. This is reduced if not eliminated with OTTLE experiments, suitable therefore for spectral characterization of the products of electrode reactions (ref. 16). A variation 
of the optically transparent electrode is the combination of a rotating disc electrode with an optically transparent ring for spectrophotometric investigations of intermediates in complex electrochemical processes (ref. 17).

Investigation of thick films: Special problems arise with thicker films.

- Multiple reflections at the film boundaries cause interference, the typical effect being a periodic increase and decrease of transmittance or absorptance at constant wavelength with growing thickness, or at constant thickness with changing wavelength. Although the first transmission extremum appears at a film thickness of $\lambda / 4 n$ ( $n=$ refractive index of the film), already a film thickness less than $\lambda / 4 n$ causes deviations from the Lambert-Beer law. Calculation of the optical constants of the film is recommended.

- A second problem is nonuniformity and roughness of the film. Several attempts have been made to take this problem into account (e.g. ref. 18).

Comments: Ideally the absorption coefficient of the reactant (substrate) should be low in the wavelength range of the absorption maxima of the products. Extraction of spectra and estimation of homogeneous rate constants for chemical reactions following electron transfer are model (mechanism) dependent. For a first-order chemical reaction following electron transfer, rate constants up to $5 \times 10^{4} \mathrm{~s}^{-1}$ can be measured, assuming an absorption coefficient of the product of $10^{4}$ and an absorbance sensitivity of $10^{-}$ 4.

\subsection{Long Optical Path Thin Layer Cells}

In long optical path thin layer cells, LOPTLC, the optical beam is parallel to the electrode surface (Fig. 3 , ref. 19). The width of the thin layers is of the order of 1 to $1000 \mu \mathrm{m}$, with a pathlength from 1 to 10 $\mathrm{cm}$. If the potential is changed by steps, the absorbance at each potential is measured after a homogeneous distribution of solution species in the thin layer has been reached. The recommended units for representing the data are the same as for optically transparent electrodes: absorptance, $\alpha(E)$, and differential absorptance, $\Delta \alpha(E)=\alpha(E)-\alpha\left(E_{0}\right)$, versus potential, wavelength or time. The electrode can be opaque. Lasers can be used to avoid scattering losses at the electrode surface. By exhaustive electrolysis ( $2 \mathrm{~min}$, see ref. 18) intermediates can be detected (if their lifetimes are sufficiently long, 5-10 min).

The cell can be used to investigate electroadsorption, which can be measured by the concentration change, due to the small cell volume (ref. 19).

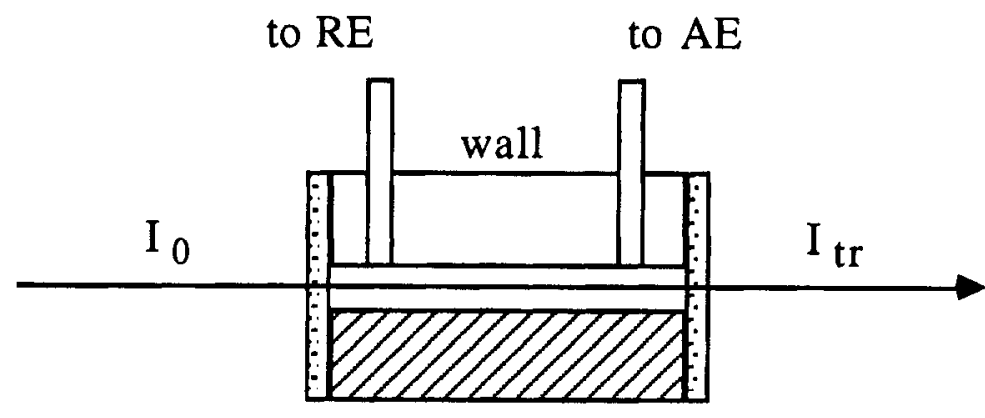

$\mathrm{WE}$

Fig. 3. Long optical path thin layer cell, LOPTLC. $I_{0}, I_{\text {tr }}$ incident, transmitted intensity, respectively; WE, working electrode, AE, auxiliary electrode and RE, reference electrode are located outside the thin layer area.

Comments: As the optical path is longer than in OTEs, species with lower absorption coefficients can be 
measured. As the volume is small, the technique is useful for electroadsorption studies.

\subsection{Diffusion Layer Imaging (or Profiling)}

This technique allows to obtain directly absorbance vs. (distance from the electrode) profiles by means of a collimated laser beam parallel to the electrode, with a diameter of a few hundreds of micrometres, which is then magnified and imaged onto a photodiode array. There is a linear relationship between the position of a detector in the array and the distance from the electrode. It is necessary to use lasers whose frequency is at or near the absorption maximum of the electrogenerated or electroconsumed species, which allows to obtain directly the corresponding concentration profile by application of the BeerLambert's law.

The technique was designated as Diffusion Layer Imaging by its inventors $(20,21)$. The name Diffusion Layer Profiling would be more descriptive, and in line with the name "Depth Profiling" commonly used in Surface Science.

\section{REFLECTANCE EXPERIMENTS}

\subsection{UV/VIS Reflectance Spectroscopy}

In UV/visible reflectance spectroscopy, the classical theory of reflection at a solid-liquid interface in the presence of a surface layer allows the calculation of the absorption spectrum of the layer (section 2), a three-layer model being appropriate in many cases. For very thin layers the linearized equations of the Fresnel reflection coefficient can be used.

\subsubsection{UV/VIS Differential Reflectance Spectroscopy}

The scheme of reflectance measurements is shown in Fig. 1. The intensity of the reflected beam is measured as a function of potential, and division by the intensity of the incident beam gives the reflectance, $\rho(E)=I_{\text {refl }}(E) / I_{0}$. The reflectance is measured at two potentials, a reference potential, ideally one at which the surface is film-free, and a potential at which a surface film (for example, an oxide or metal film) is formed. The normalized differential reflectance, $\Delta \rho(E) / \rho(E)$, is plotted versus potential or wavelength (ref. 22).

Differential reflectance spectroscopy is useful for obtaining absorption spectra of molecular films as a function of the redox state of the film, which depends on the electrode potential. Dye films with high enough absorption coefficients can be studied down to less than monolayer thickness.

Since the signal-to-noise ratio increases with the square root of the number of spectra accumulated, an ingenious rapid scanning spectrometer was developed (ref. 23), but it has been long superseded by photodiode or CCD arrays (optical multichannel analyzers (OMAs)), with which a whole spectrum can be collected in a few milliseconds. Data presentation should include the number of scans integrated and the integration time.

Comments: This method is suitable for investigation of multilayer films of oxides or metals, or monolayers of strongly absorbing molecules, atoms or ions. The method is also preferred for investigations at surfaces of single crystals. Sensitivity: $\Delta \rho / \rho<10^{-4}$.

\subsubsection{UVNIS Electrochemically Modulated Reflectance Spectroscopy or Electrolyte Electroreflectance Spectroscopy}

Reflectance sensitivity is increased if the potential is modulated and the modulation of the reflected light detected using a lock-in amplifier technique. The method is frequently abbreviated as EMRS. Another name used in the literature is "potential modulated reflectance spectroscopy" (PMRS). Actually, EMRS is the application to electrochemistry (e.g. ref. 24,25) of a technique developed by physicists and designated by them as Electrolyte Electroreflectance (EER, ref. 26,27) or just Electroreflectance Spectroscopy (ERS). 
Measurements are usually made for two states of polarization of light, perpendicular, s, and parallel, $\mathrm{p}$, to the plane of incidence. Modulation is achieved by superimposing an alternating voltage on a stationary or slowly scanned electrode potential. Typical modulation conditions are: frequency, 10-100 $\mathrm{Hz}$; peak-to-peak amplitude, $(\Delta U): 100 \mathrm{mV}$. The modulation of the intensity of the reflected beam $\Delta I$ is detected by a lock-in amplifier. Dividing $\Delta I$ by the mean intensity of the reflected beam, $I$ and by the modulation amplitude of the potential, $\Delta U$, yields the normalized modulated reflectance modulation, $(1 / \rho) \Delta \rho / \Delta U$, which may be plotted versus potential or wavelength.

Modulation of the potential can change the interfacial charge and/or the chemical composition of the interface. The term Electroreflectance Spectroscopy (ERS) or, more precisely, Electrolyte Electroreflectance Spectroscopy (EERS) should be reserved for the first case, and Electrochemically Modulated Reflectance Spectroscopy (EMRS) for the second case. It is not always possible to unequivocally distinguish between the two possible origins of a modulated reflectance signal.

ERS is used for determining the energies of the characteristic points in the band structure of semiconductors, and also for investigating the optical properties of metal electrodes (27).

ERS was first applied in 1973 for the study of corrosion layers on metals (28). Its very high sensitivity, and the reasonable assumption that usually the electroreflectance spectra show structure only at the frequencies of the absorption edges and bandgaps of the compounds in the passive fim (29), render ERS a very useful technique for corrosion studies.

Electrochemically Modulated Reflectance Spectroscopy (EMRS) is a versatile technique which has been used for the unequivocal detection of reation intermediates (30), the unequivocal distinction between the ECE and disproportionation mechanisms (31), the electrochromism of organic molecules (31a), the potential dependence of the acidity constant of species in the double layer ( $31 \mathrm{~b}$ ), the kinetics of the electron transfer within an adsorbed organic layer (31c), and the detection of chemisorbed $\mathrm{CO}$ (31d).

Comments: EER and EMRS are typically two orders of magnitude more sensitive than differential reflectance spectroscopy. Values of $(1 / \rho) \Delta \rho / \Delta U<10^{-6} \mathrm{~V}^{-1}$ can be measured. The modulation of the potential by $\Delta U=100 \mathrm{mV}$ causes only a moderate perturbation of the electrochemical system. The method is therefore preferred in cases where large potential changes must be avoided (e.g. studies on well defined crystallographic faces).

\subsubsection{UVNIS Wavelength Modulated Reflectance Spectroscopy}

Another reflectance spectroscopy is based on modulation of the wavelength (ref. 32), which enhances the structure of the reflectance spectrum by increasing the signal-to-noise-ratio of the reflectance without suppression of the optical contributions of the bulk phases. The modulation amplitude is not well defined (partly by instrumental restrictions) and quantitative values cannot be given. Therefore, the modulation of the intensity of the reflected beam, $\Delta I_{\text {refl }}$, is normalized by division by either the unmodulated intensity of the reflected beam or by that of the incident beam, $\Delta I_{\text {refl }} / I_{\text {refl }}$ or $\Delta I_{\text {refl }} / I_{0}=\Delta \rho$, respectively.

Comments: Because of the mentioned limitations, the method is little used, since more effective modulation methods are available.

\subsubsection{UV/VIS Photoreflectance Spectroscopy}

A periodically interrupted laser beam has been used to modulate either the electrode surface temperature (Photothermal Reflectance Spectroscopy, ref. 32,33) or the electric field at the interface, due to the generation of electron-hole pairs (Photoconductive Reflectance Spectroscopy, ref. 34).

The generated signal can be obtained only in arbitrary dimensionless units. The modulation of the intensity, $\Delta I(U)$, divided by the incident intensity, $I_{0}$, or by the reflected intensity at the same potential, $I_{\text {refl }}(U)$, is plotted as a function of potential or wavelength. $\Delta I(U) / I_{0}$ is preferred. 
Comments: This method is applied in semiconductor electrochemistry, especially to systems where electrochemical modulation fails, but it is still in an experimental stage.

\subsubsection{UVNIS Attenuated Total Reflectance Spectroscopy (Internal Reflectance Spectroscopy}

Total reflection of a light beam at a transparent electrode/electrolyte interface is achieved if the beam approaches the interface from the electrode side and at an incidence angle higher than the critical angle, $\Theta_{\text {crit }}=\arcsin \left(n_{1} / n_{3}\right)$ (Attenuated Total Reflectance (ATR) Spectroscopy, ref. 35). The transparent electrode can be a semiconductor or a thin metal film evaporated onto a transparent substrate. The name "internal reflectance spectroscopy" (IRS; ref. 36) is also used.

In ATR, total reflection of light creates an evanescent wave in the electrolyte, the penetration depth for a transparent electrolyte being (ref. 35)

$d=\frac{\lambda}{\left[4 \pi\left(n_{1}^{2} \sin ^{2} \alpha-n_{2}^{2}\right)\right]^{1 / 2}}$

ATR can be considered a variation of the thin layer technique, the spectra of electrogenerated species being similar to absorption spectra. High surface sensitivity can be achieved. Single and multiple reflectance systems have been developed (Fig. 4). The method has been used more intensively in the IR (par. 4.2.4)

Comments: ATR spectroscopy can only be applied if transparent electrodes are available. Their preparation by evaporation of a thin metal film on a transparent support is difficult and limits the choice of electrodes and their pretreatment.

\subsubsection{UVNIS Surface Plasmon Spectroscopy}

With some metals evaporated on transparent electrodes and used under ATR conditions surface plasmons (collective oscillations of surface electrons) can be excited (surface plasmon spectroscopy, ref. 37-39). The method has been used to investigate species adsorbed at metal surfaces.

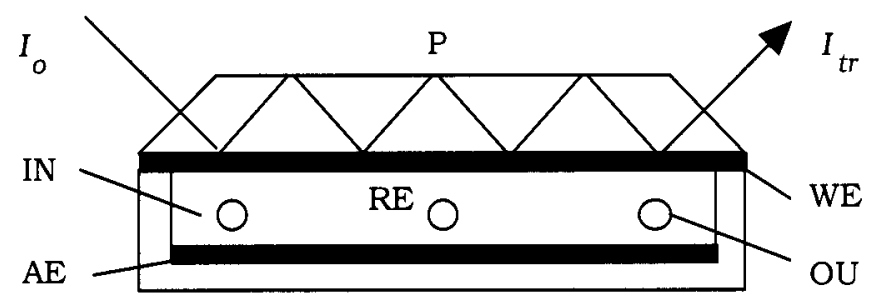

Fig. 4. Principles of attenuated total reflectance (ATR) or internal reflectance spectroscopy: $P$, prism; WE, working electrode; RE, reference electrode; $\mathrm{AE}$, auxiliary electrode; IN, electrolyte inlet; OU, electrolyte outlet; $I_{0}, I_{\mathrm{tr}}$, incident, transmitted beam.

\subsection{Infrared Reflectance Spectroscopy}

IR spectroscopy of the electrode/electrolyte interfaces requires thin layer cells (a few $\mu \mathrm{m}$ ) because IR radiation is strongly absorbed by the electrolyte and/or solvent. Two approaches have been followed: external reflection (irradiation through the electrolyte layer) using a thin layer cell, and internal reflection (irradiation through a transparent electrode at an incidence angle higher than the critical angle) utilizing the thin penetration zone (evanescent wave) of the electromagnetic field in the electrolyte upon total reflection. 
In the infrared region only electric fields perpendicular to the surface can exist on metals. This is the basis of the "Surface Selection Rule", which states that an adsorbed molecule can absorb energy from an infrared beam only if the dipole moment of the molecule is perpendicular to the metal surface.

The extensive development of Fourier Transform Infrared (FTIR) spectrometers has stimulated the use of this technique in electrochemistry.

\subsubsection{Electrochemically Modulated Infrared Reflectance Spectroscopy}

This method is usually abbreviated as EMIRS. The electrode potential is modulated between two values, $U_{1}$ and $U_{2}$, by a square wave signal, the modulation amplitude, $\Delta U=U_{1}-U_{2}$, varying between 50 and $1000 \mathrm{mV}$. A low modulation frequency is used (typically between 5 and $20 \mathrm{~Hz}$ ). The relative change of the reflectance, $\Delta \rho / \rho$ where $\rho$ is the reflectance at the mean potential $U=\left(U_{1}-U_{2}\right) / 2$, is plotted versus the wavenumber, $v$. Several spectra are recorded as a function of the potential, $U$. The sensitivity achieved by this technique is $\Delta \rho / \rho<10^{-5}$ (ref. 40).

Organic adsorbates give rise in EMIRS to a spectrum which is the pseudo-first derivative of the absorption spectrum, due to an electroreflectance phenomenon, namely a shift of the band position with electrode potential (Stark shift, which is about $30 \mathrm{~cm}^{-1} / \mathrm{V}$ for chemisorbed $\mathrm{CO}$ ).

In a variant of EMIRS the bias potential is scanned at a constant modulation amplitude and wavelength. This technique suffers from severe signal-to-noise problems (ref. 41).

Comments: This is a sensitive technique but has been extensively used by only one laboratory.

\subsubsection{Differential Fourier Transform Infrared Reflectance Spectroscopy}

FTIR-spectrometers are single-beam instruments, and therefore a reference spectrum is needed in order to obtain a differential spectrum.

In the simplest case, the difference between the spectrum at a measurement potential and the spectrum at a reference potential is obtained, up to 1000 scans being collected at each potential in order to increase the signal-to-noise ratio. With the older instruments this required a long accumulation time, which led to a considerable drift of the base line. In order to compensate for this drift, a series of e.g. 50 scans were collected alternately at the measurement and reference potential, until the desired number of scans at each potential had been carried out, after which all the scans collected at each of the two potentials were separately co-added. This technique was denominated SNIFTIRS (Subtractively Normalized Interfacial FTIRS, ref. 42), an acronym which makes no reference to the alternating character of the collection of scans. The denomination Square Wave-FTIRS (SW-FTIRS) should be preferred

An increasingly used FTIRS technique consists of collecting scans continuously during a Linear Potential Sweep (LPS), the interferograms collected during consecutive equal time intervals being stored separately. Differential spectra are obtained by subtracting from each spectrum a reference spectrum, usually that recorded at the highest or lowest potential. The acronym SPAIRS (Single Potential Alteration IRS) was coined for this technique, but its use should be discouraged. The more descriptive name Linear Potential Sweep FTIRS (LPS-FTIRS) should be preferred.

Other technique increasingly used in electrochemical FTIRS consists of collecting e.g. 50 scans at a series of different potentials, following any pattern thought to be convenient, after which the spectrum at any of the potentials can be subtracted from that at any other potential. Then the generic name Potential Difference FTIRS (PDFTIRS) should be used.

Comments: The sensitivity of FTIRS is usually lower than that of EMIRS, but increases with the accumulation time. $\Delta \rho / \rho<10^{-4}$ can be considered a typical value for medium accumulation times (several hundred interferograms). Discrimination between changes of surface concentration and changes in concentration in the diffusion layer requires the use of both $\mathrm{p}$ and $\mathrm{s}$ polarization, taking advantage of the surface selection rule. This problem is less pronounced in EMIRS, where only those changes which can follow the modulation frequency $(\mathrm{ca} .10 \mathrm{~Hz}$ ) are measured. 


\subsubsection{Polarization Modulated Fourier Transform Infrared Reflectance Spectroscopy.}

In this technique (abbreviated as PMFTIRS), Fourier transform spectroscopy is combined with polarization modulation, typically with a $\mathrm{ZnSe}$ photoelastic modulator at $74 \mathrm{kHz}$. According to the "surface selection rule", only adsorbed molecules with the dipole moment perpendicular to the surface absorb IR energy. Therefore, modulating the state of polarization should yield the IR spectrum of adsorbed species, since contributions from the solution and the diffusion layer are eliminated by this technique. However, solvent molecules will also appear in PMFTIRS, regardless of their orientation with respect to the electrode surface. Further $\mathrm{S} / \mathrm{N}$ improvement is obtained if a spectrum at a reference potential can be subtracted (ref. 43).

This method has also been used with dispersive instruments (polarization modulated (dispersive) infrared reflectance spectroscopy, PMIRS) (ref. 44).

Comments: The gain in sensitivity $\left(\Delta \rho / \rho<10^{-5}\right)$ must be weighed against the technical difficulties. The modulation frequency must be of the order of $100 \mathrm{kHz}$ to exclude interference from the FTIR interferometer. This is a sensitive method applicable to adsorbed molecules only.

\subsubsection{Multiple Internal Reflection Fourier Transform Infrared Reflectance Spectroscopy}

In ATR-FTIR spectroscopy usually the multiple reflection mode is used (ref. 45.46). FTIR reflectance spectra are collected and averaged as the difference between the spectrum at a potential, $U$, and a reference potential, $U_{0} . \Delta \rho / \rho$ versus wavenumber, $v$, is usually plotted as a function of the potential, $U$. The method is less sensitive than SW-FTIRS (SNIFTIRS).

Comments: Electrode surface films (passivating oxide films, films of conducting polymers, etc.) were investigated by this method, special advantages obtaining with transparent semiconductor electrodes.

\section{METHODS BASED ON THE POLARIZATION OF LIGHT}

\subsection{Ellipsometry}

In ellipsometry, the ratio of the complex Fresnel reflection coefficient for parallel polarization to that for perpendicular polarization, $\hat{\rho}$, is measured (ref. 4,47,48). This complex quantity, $\hat{\rho}$, should be distinguished from the reflectance, $\rho$, a real quantity, and is given by

$$
\hat{\rho}=\tan \Psi \exp (\mathrm{i} \Delta)
$$

where $\Psi=\arctan r_{\mathrm{p}} / r_{\mathrm{s}}{ }_{(} r_{\mathrm{p}}, r_{\mathrm{s}}=$ amplitudes of the Fresnel reflection coefficients for $\mathrm{p}$ - and $\mathrm{s}$ polarized light, respectively), and $\Delta=\delta_{\mathrm{p}}-\delta_{\mathrm{S}}, \delta_{\mathrm{p}}, \delta_{\mathrm{S}}=$ phase change upon reflection for $\mathrm{p}$ - and spolarized light, respectively). It is easy to determine the complex refractive index of a flat absorbing substrate in contact with vacuum or with an electrolyte of known refractive index, since there are two unknowns and two experimental parameters. However, if an absorbing electrode of known complex refractive index, covered with an absorbing film of unknown complex refractive index and unknown thickness, is in contact with an electrolyte of known refractive index, a further parameter besides $\Psi$ and $\Delta$ is required for determining the three unknowns of the surface layer. In this case several approaches have been used, involving the measurement of i) $\Delta, \Psi$ and the reflectivity of unpolarized light (threeparameter ellipsometry, ref. 49-51); ii) the reflectivity of polarized incident light at three azimuths of polarization of the reflected light (ellipso-reflectometry, ref. 52); and iii) the reflectivities of incident $\mathbf{s}$ and p polarized light at several angles of incidence (multiple angle of incidence reflectometry, ref. 53). Ellipsometric data are easier to evaluate for very thin films, for which the linear approximation can be applied (ref. 54), but linearization can be dangerous.

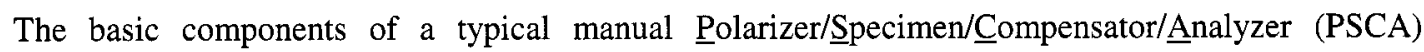
ellipsometer are illustrated in Fig. 5. Light from the source L passes through the polarizer P, where it is 
linearly polarized at a given angle to the plane of incidence, i.e. with the parallel (p) and the perpendicular (s) components of equal phase but unequal amplitude. The linearly polarized light is then reflected by the electrode, whereupon it becomes elliptically polarized. The azimuthal orientation of the polarizer is chosen such that the $\mathrm{p}$ - and s-components of the reflected light have the same amplitude. The compensator, $\mathrm{C}$ (a quarter-wave plate), with its fast axis oriented at $45^{\circ}$ to the plane of incidence, turns back the elliptical polarization into linear polarization by compensating the phase difference, $\Delta$. The azimuth of orientation of the analyzer, $\mathrm{A}$, is adjusted so that the light intensity at the detector is at a minimum. In this "nulling" mode of operation $\Psi$ and $\Delta$ are determined by the orientation of $\mathrm{P}$ and A, respectively, required to give a minimum intensity at the detector. Automation of the nulling ellipsometer can be attained.

Two-parameter ( $\Delta$ and $\Psi$ ) ellipsometry is restricted to transparent films like $\mathrm{SiO}_{2}$. For absorbing films, usually three-parameter ellipsometry (ellipso-reflectometry) is chosen (ref. 49-53). A compensator is not required in some types of automatic ellipsometers, e.g. rotating analyzer ellipsometers, which are well suited for spectroscopic ellipsometry.

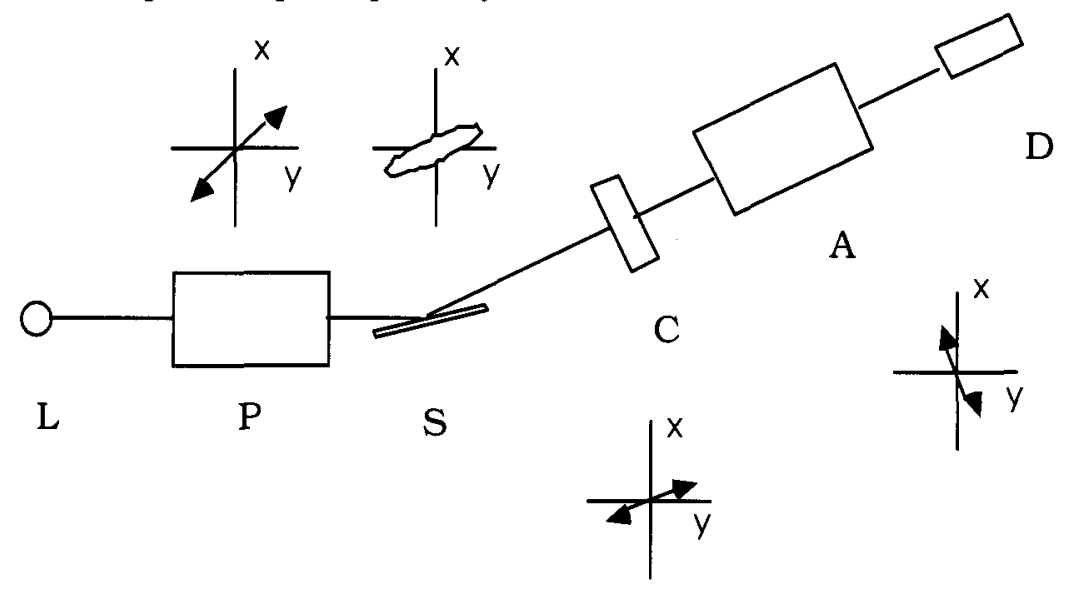

Fig. 5. The PSCA-arrangement for ellipsometry: L, monochromatic light source; P, polarizer; S, sample; C, compensator; A, analyzer; $\mathrm{D}$, detector.

Ellipsometric results can be presented in several ways:

- as plots of $\Delta$ versus $\Psi$

- $\quad$ in the form $\Psi=f(U)$ and $\Delta=f(U)$;

- $\quad$ in the case of spectroscopic ellipsometry, as $\Psi=f(\lambda)$ and $\Delta=f(\lambda)$.

- the calculated optical constants, $\varepsilon^{\prime}, \varepsilon^{\prime \prime}$ or $n, k$ can be also given as a function of electrode potential $U$

Data analysis requires a surface model with one or more surface layers, which can be dangerous because of the limited number of independent experimental parameters. When the ellipsometric results are represented by the raw data of $\Delta$ and $\Psi$ (and reflectance), the angle of incidence used in the measurements should be stated explicitly for the raw data to have quantitative significance.

Comments: Ellipsometry is the method of choice for determining the optical constants of a bulk phase or the refractive index and thickness of a transparent surface film. Frequently, the method is only used for the measurement of the film thickness, disregarding other information. For the evaluation of data, especially the calculation of film thickness, fully computerized ellipsometers are available, often with the capability of three-parameter operation 


\subsection{Circular and Linear Dichroism}

Recently the circular or linear dichroism of a surface film has been measured. The method is a special case of spectroscopic ellipsometry, with restriction to the frequency dependence of the rotation of the plane of the electromagnetic wave (ref. 54,55).

Comments: The method may become important for polymer films, both insulating and conducting, and for biomolecules.

\section{METHODS BASED ON SCATTERED LIGHT}

\subsection{Light Scattering from Non-flat Surfaces}

Light scattering can be used to characterize the growth of nuclei on flat electrodes during the electrodeposition of metals (ref. 57). Light scattering by surface irregularities provides a statistical description of the roughness amplitude and of the spatial periodicity of light scattering centers (ref. $57,58)$.

UV/VIS diffuse reflectance spectroscopy can be used to detect the formation of light-scattering porous corrosion films a few monolayers thick (ref. 59).

Comments: These methods provide a fast in situ statistical imaging method intermediate between visual microscopy and atomic resolution (STM or AFM) microscopy.

\subsection{Surface Raman Spectroscopy}

This method is based on the scattering of a laser by interaction with vibrational modes of surface layers (phonons) or of molecules in the surface region (ref. 60). The spectra are generally measured at wavelengths longer (lower energy) than the excitation wavelength (Stokes region), the horizontal axis being the difference in wavenumbers between the excitation and scattered radiation. If photon-counting detection is used, the intensity is usually given in counts/s and should, but often is not, be corrected for the spectral sensitivity of the detection optics. When charge-coupled devices (CCD) are employed as detectors, the integration time and number of registered spectra, as well as other details of $S / N$ improvement, should be provided.

With sophisticated optical and electronic techniques spectra of monolayers of adsorbates can be obtained (ref. 61). The technique requires not only the use of very sensitive detectors (CCD) and signal averaging systems, but also special arrangements for collecting the scattered light (elliptical mirrors, superfast optics, etc.).

Comments: This is a very convenient method for obtaining, without the thin-layer limitation of infrared spectroscopy, in situ vibrational spectra of surface layers and surface films with $d>10 \mathrm{~nm}$, if intense laser illumination can be tolerated. Damage of the sample by the laser beam can be decreased by rotating the sample. Thicker oxide or other films give Raman signals that can be measured with normal technology. The technique is applicable to monomolecular films only if sophisticated light collecting systems and electronics are available and extended laser exposure can be tolerated.

\subsubsection{Surface Enhanced Raman Spectroscopy}

The SERS acronym is well established in the literature. Some metals (particularly $\mathrm{Ag}, \mathrm{Cu}$, and $\mathrm{Au}$ ) show surface enhancement of Raman scattering (ref. 62,63), which is observed on micro (nano) rough surfaces, the roughness being usually produced by a previous potential cycling. Therefore, the method is not applicable to single crystals. Several theories partially explain the enhancement effect (the electromagnetic (EM) theory (ref. 64), the charge transfer (CT) theory (ref. 65), etc.). Surface enhancement can also be achieved by electrodeposition, or by coating an adsorbate with colloidal or deposited Ag particles, which makes possible Raman spectroscopy of surfaces which do not ordinarily 
show such enhancement (e.g., glassy carbon). Excitation is thus far restricted to the visible region. With excitation in other frequency domains, other metals will show the enhancement effect (ref. 66)

Comments: This method is usually restricted to $\mathrm{Ag}, \mathrm{Cu}$ and $\mathrm{Au}$.

\subsection{Nonlinear Optical Techniques}

Two nonlinear optical techniques are increasingly used, Second Harmonic Generation (SHG) and SumFrequency Generation (SFG). In SHG a fraction of the light of a pulsed laser (e.g., a Neodymium-YAG laser), is doubled in frequency upon scattering from a surface (Fig. 6, ref. 67), in a typical nonlinear optical phenomenon. The advantage of the method is the dependence of the scattering intensity on the two-dimensional crystallographic symmetry of the electrode surface, which allows the detection of surface reconstruction and to determine the orientation of adsorbed molecules. The scattered intensity is measured as a function of the electrode potential. To obtain the two-dimensional surface symmetry, the scattered intensity at a fixed potential is measured as a function of the angle of rotation of the electrode surface and the plane of polarization of the exciting laser beam.

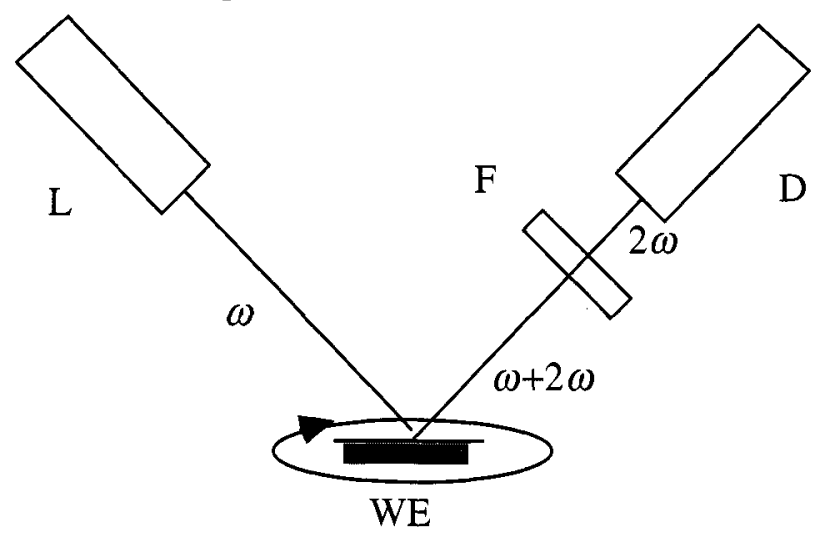

Fig. 6. Scheme of second harmonic generation: L, laser; WE, working electrode; F, filter allowing to pass light of frequency $2 \omega$; only; $\mathrm{D}$, detector.

In SFG the second-harmonic output (of frequency $\omega_{\text {vis }}$ ) of a laser, and a tunable IR laser (of frequency $\omega_{\text {ir }}$ ) from an optical parametric amplifier, are focused to a common focal spot on the interface to be studied, and the sum-frequency signal (of frequency $\omega_{\mathrm{vis}}+\omega_{\mathrm{ir}}$ ) is detected (ref. 68)

Comments: SHG can be used to study the surface geometry of single crystals and its potential dependence.

\section{X-RAY AND $\Gamma$-RAY TECHNIQUES}

Reflection and diffraction of mainly synchrotron radiation in the X- and $\gamma$-ray spectral region can be used for in situ electrochemical studies if the electrolyte layer is sufficiently thin to transmit adequate beam intensity, and if the $\mathrm{S} / \mathrm{N}$ ratio allows discrimination between the bulk and surface layers. X-rays are, in principle, not surface sensitive because of their large penetration depth (except for total external reflection). After first reports with conventional X-ray sources (ref. 69), real progress started when synchrotron radiation became available (ref. 70-72).

\subsection{X-ray diffraction (XRD)}

The study of the atomic structure of thick crystalline oxide films or electrodeposited metal layers $(d>$ $100 \mathrm{~nm}$ ) may be performed with conventional X-ray sources. Information is obtained by application of 
the Bragg's equation, $d_{h k l}=\lambda / 2 \sin \Theta$. Thinner films down to laterally ordered monoatomic adsorption layers require intensive synchrotron light sources, preferentially very intense radiation of wiggler or undulator beam lines. In order to enhance the contribution of surface films to the diffraction pattern amd to suppress those of the bulk material of the working electrode a grazing incidende geometry of the X-ray beam is necessary. Diffraction spots are measured as usual. The evaluation of the diffraction pattern yields the crystal structure of ordered surface layers. The termination of a crystal at the surface causes crystal truncation rods (CTR) with a characteristic change of the X-ray intensity between the Bragg's reflections which contains valuable information about the structural deviation of surface layers from the bulk. Reconstructed surface layers and adsorption layers yield additional truncation rods which are lower in intensity by a factor of $10^{-6}$.

\subsection{X-Ray Absorption Spectroscopy (XAS)}

Absorption spectra are measured using a crystal monochromator on the exit of the beam line or with polychromatic light with a position sensitive detector. The region of the spectrum close to the absorption edge of an element, i.e. the near edge X-ray absorption spectrum (NEXAFS) or the X-ray absorption near edge spectroscopy (XANES) yields valuable information on the valence state and the chemical environment of the absorbing atom. The extended X-ray absorption fine structure (EXAFS) contains information about the near range order of crystalline, disordered or even amorphous materials. EXAFS is the result of an interference process. X-ray absorption leads to the ionisation of core levels of the atoms of the specimen. The outgoing eletron wave or the photoexcited electron interferes with the electron wave reflected at the various coordination spheres close to the absorber atom. This interference process modulates the absorption of the X-rays with energies larger than that of the absorption edge by up to several $100 \mathrm{eV}$. The Fourier analysis of this modulation separates the influence of the contributing coordination spheres and yields structural data, such as the distance and the coordination numbers of the coordination shells and their structural and thermal disorder by the Debye-Waller factor. The specimen may contain more than one kind of species with sufficiently high energies of the absorption edge. In these cases one may analyse the atomic near range order for both of the different absorbing species which are complementary to each other.

XAS is a bulk method which is used conventionally in the transmission mode. It permits electrochemical in situ studies of the structure of thick layers on electrodes and its change during electrochemical or chemical reactions. Structural changes of clusters in a conducting matrix can be determined. The strength of XAS in transmission lies in the examination of catalytic processes in gaseous and electrolytic environment. Structural changes of even disordered or amorphous materials are accessible. Processes on flat electrode surfaces may be studied with XAS in reflection using a grazing incident X-ray beam. The absorption spectrum is obtained by measuring the specularly reflected beam or the fluorescence radiation. Surface sensitivity is achieved if the angle of incidence is lower than the critical angle for total reflection. The measured modulated reflectivity may be compared to the computer simulated data of appropriate standards, applying a Kramers-Kronig transform to the components of the complex refraction index and Fresnel's theory. This evaluation procedure on a comparative basis yields the data for the near range order of surface layers. Suitable designs for the electrochemical cells minimize the serious absorption of X-rays within the electrolyte. The importance of absorption losses decreases with increasing energy of the X-rays. XAS studies are possible for copper and heavier elements, i.e. with energies $<9 \mathrm{keV}$.

Comments: These methods suffered from severe intensity problems, but have gained increasing importance with availability of synchrotron radiation sources of high luminosity.

\subsection{Mößbauer Spectroscopy}

Some metals with radioactive isotopes which emit $\gamma$-radiation of suitable characteristics can be investigated by Mößbauer-spectroscopy; ${ }^{57} \mathrm{Fe}$ is the most prominent example (ref. 73). Commercial 
spectrometers are available. Integration times are extremely long, even for thicker films. Corrosion layers can be investigated, but the passive film itself is too thin to give reliable signals unless ${ }^{57} \mathrm{Fe}$-enriched iron and/or conversion-electron techniques (CEMS or DCEMS) are used.

Comments: The method requires long accumulation times (hours to days). It is restricted to elements having a suitable $\gamma$-emitting isotope, but it is a valuable method in special research areas like corrosion, etc.

\section{MAGNETIC RESONANCE METHODS, MICROWAVE SPECTROSCOPY}

\subsection{Electron Paramagnetic Resonance (EPR, ESR)}

If electron transfer in electrochemical reactions leads to the formation or disappearance of paramagnetic species, these species can be characterized by electron spin resonance spectroscopy. The total energy of a free electron in a magnetic field of strength $\mathrm{H}$ is split into two terms according to

$$
E=E_{0} \pm \frac{1}{2} g \mu_{\mathrm{B}} B
$$

with $\mathrm{g}$, the g-factor, $\mu_{\mathrm{B}}$, the Bohr magneton and $B=\mu H$, the magnetic induction. Irradiation of the system with photons of energy $g \mu_{\mathrm{B}} B$ will cause resonance absorption. Microwaves of the X-band range (about $9.6 \mathrm{GHz}$, corresponding to centimetre waves) are required for the usual magnetic fields of about $3300 \mathrm{G}$. The wave guides and the special resonator cause some restrictions in electrochemical cells used for in situ measurements (ref. 74). To minimize microwave absorption in the electrolyte solution a thinlayer cell is required, which causes the same problems as in optical spectroscopy (see par.3.1). New developments in cell design overcome this problem.

ESR-spectroscopic detection of electrochemically produced paramagnetic species was first realized in 1958 (ref. 75) and is nowadays widely used as a standard method in electrochemical research (ref. 7680). The main field of application is the qualitative description of the structure, determining the spin distribution in the molecules by using hyperfine splitting constants and simulation of the ESR-spectra. The kinetic analysis of radical formation and decay is possible down to radical lifetimes as low as about $50 \mathrm{~ms}$ (ref. 81). The detection of paramagnetic species is not restricted to electrolyte solutions, but also applies to solid electrode materials like conducting polymers, carbon, and others. Recently the spin-trap and the spin-label (spin probe) techniques have been used in electrochemical ESR spectroscopy.

\subsection{Microwave Absorption}

Photogenerated charge carriers in a semiconducting electrode placed in a waveguide sytem will absorb microwave radiation. The relative change of microwave power reflected from the illuminated semiconducting electrode is proportional to the integral over all minority carriers in the illuminated surface (ref. 82).

\subsection{Nuclear Magnetic Resonance (NMR) Spectroscopy}

Due to the fact that most elements have an isotope with finite nuclear spin, the applicability of nuclear spin resonance spectroscopy is much broader than that of ESR-spectroscopy. Thus, NMR-spectroscopy is used as a standard ex situ method for the analysis of reaction products, especially in organic electrochemistry. However, there are only a few in situ studies of NMR-spectroscopy due to its low sensitivity. Some solid-state NMR applications show great promise (ref. 82a)

Comments: EPR is useful for characterizing paramagnetic species in solution. If the spectra are complicated (hyperfine splitting), or if a mixture of species is produced, higher concentrations or longer lifetimes are required. Species on the electrode surface cannot be detected by EPR. 
Both the technique and the theory of microwave absorption are still in an early stage.

\section{PHOTOCURRENT AND PHOTOPOTENTIAL SPECTROSCOPIES}

If a semiconductor electrode is illuminated by light of an energy higher than the band gap, photoelectrons and photoholes are generated within the space-charge layer. The field gradient in this layer causes a separation of the positive and negative charge carriers, and so photocurrents or photopotentials can be measured. The principles of semiconductor photoelectrochemistry were covered by a recent IUPAC paper (ref. 83). At lower light intensities the photosignals are proportional to the absorbed intensity. The photocurrent or the photopotential are plotted as a function of the potential or the wavelength, in the latter case the wavelength dependence reflecting the absorption spectrum (ref. 84). Lasers can be used as the light source (laser photoelectrochemistry).

If molecules are adsorbed on the semiconductor or oxide surface, electrons can be excited from the valence band into unoccupied orbitals of the molecules, or from occupied orbitals of the molecules into the conduction band. Again a photocurrent is observed, and can be plotted versus potential or wavelength. The wavelength dependence is proportional to the absorption spectrum of the adsorbed molecules (ref. 85, 86).

With light pulses or pulsed lasers, the kinetics of recombination processes can be studied. Using a modulated light source in combination with phase-sensitive detection, the photocurrents in-phase and $90^{\circ}$ out-of-phase can be measured, as in impedance spectroscopy. This method is called intensitymodulated photocurrent spectroscopy (IMPS, ref. 87, 88).

In metals a photocurrent can be observed if electrons emitted from an illuminated electrode become solvated and react with scavenger molecules in the electrolyte (ref. 89, 90). With high-intensity pulsed lasers the kinetics of very fast processes can be measured. The temperature jump in the area illuminated by the laser pulse has also been used for kinetic studies (ref. 91).

Comments: Photocurrent and photopotential measurements offer a wide variety of possible spectroscopic applications. The simple experimental setup is a convenient gate to spectroelectrochemistry.

\section{PHOTOTHERMAL METHODS}

Periodic illumination of a thin electrode at a wavelength at which it absorbs will heat it. The increase in temperature can be detected either with a microphone or with a piezoelectric transducer, in which the heat-induce stress generates an electric signal. Measuring the signal as a function of wavelength gives the absorption spectrum of the electrode material. Potential-induced changes in this spectrum can be investigated (ref. 92-96). The method has been used with both metal and semiconductor electrodes. Although the name Photoacoustic Spectroscopy is well established, a more descriptive name would be Photothermal Spectroscopy.

\subsection{Photothermal Probe Beam Deflection Spectroscopy}

This technique has also been designated as Mirage Spectroscopy (96a) and Transverse Photothermal Deflection Spectroscopy (96b). The changes in the refractive index of the electrolyte produced by the absorption by the electrode of a light beam perpendicular to the electrode surface are monitored by the deflection of a laser beam parallel to the electrode surface and impinging on a position-sensitive detector (photodiode array). The changes in refractive index are due to changes in electrolyte temperature, and not in the concentration of some species. We propose for this technique the name Photothermal Probe Beam Deflection Spectroscopy, and the acronym PPDS. In the solid-liquid interface it is two orders of magnitude more sensitive than Photoacoustic Spectroscopy (96c). 


\subsection{Probe Beam Deflectometry}

With a laser beam of small diameter, parallel and very near the electrode surface, concentration gradients can be detected, since the corresponding changes in the refractive index of the solution will deflect the laser beam. A position sensor (photodiode array) allows the detection of minute deflections of the laser beam. The technique is known as Probe Beam Deflection Spectroscopy, abbreviated as PDS. Actually the name Probe Beam Deflectometry (abbreviated as PBD) should be preferred, since no quantum absorption is involved. A plot of the probe beam deflection vs. potential recorded during a cyclic voltammogram is known as a cyclic deflectogram (96d).

\section{LIGHT EMISSION METHODS}

Electrochemical reactions can generate light emission. The spectrum of the emitted light can yield important information about the electrode process and the state of the electrochemical system.

\subsection{Electrochemical Luminescence}

The substantial energies involved in the reaction of electrochemically generated strong oxidants and reductants often leads (by a variety of mechanistic paths) to excited-state reaction products, which may show luminescence (electrochemical luminescence, ECL), yielding a spectrum similar to the fluorescence spectrum of the reaction products (ref. 97). In addition to organic radical ions, transition metal complexes have also been studied.

Intermediates which undergo homogeneous reactions might also cause light emission.

\subsection{Electroluminescence of Semiconductors}

Electrolysis at semiconductor electrodes can produce light emission when electrons from species in the solution are injected into empty energy levels of the conduction band and recombine with holes in the valence band (p-type semiconductor), or when holes from species in the solution are injected into the valence band and recombine with electrons in the conduction band (n-type semiconductor). The emission spectrum follows the absorption spectrum of the semiconductor. Crystallographic imperfections acting as recombination centers, traps, or surface states, influence the emitted intensity and can therefore be detected with this spectroscopy (ref.98-100).

Comments: The use of light emission measurements (ECL, EL of semiconductors) is restricted to a few laboratories, since very few systems are amenable to this method. 